
\title{
25 Research Soure \\ The role of Trx/TBP-2 in oxidative stress induced autophagy in human lens epithelial cells
}

Jianghua Hu

Zhejiang University School of Medicine Second Affiliated Hospital https://orcid.org/0000-0002-62171257

\section{Lifang Shen}

Zhejiang University School of Medicine

\section{Chengshou Zhang}

Zhejiang University School of Medicine Second Affiliated Hospital

Ke Yao

Zhejiang University School of Medicine Second Affiliated Hospital

Yibo Yu ( $\nabla$ yuyibo@zju.edu.cn )

https://orcid.org/0000-0001-9256-4687

\section{Research article}

Keywords: Cataract; Oxidative Stress; Autophagy; Thioredoxin; Thioredoxin binding protein-2

Posted Date: November 27th, 2019

DOl: https://doi.org/10.21203/rs.2.17897/v1

License: (c) (i) This work is licensed under a Creative Commons Attribution 4.0 International License.

Read Full License 


\section{Abstract}

Background: Oxidative stress plays an important role in age-related cataract development. The cellular antioxidant protein thioredoxin (Trx) and its negative regulator, thioredoxin binding protein-2 (TBP-2), maintain the intracellular redox balance upon oxidative stress. The aim of this study is to investigate role of Trx and TBP-2 in human lens epithelial cells (LECs) under oxidative stress. Methods: LECs were treated with $50 \mu \mathrm{M}$ of $\mathrm{H} 2 \mathrm{O} 2$ serum-free medium for different duration, and the mRNA and protein levels of Trx-1, Trx-2 and TBP-2 were measured by reverse transcription-polymerase chain reaction and Western blot. Trx1 activity was evaluated by Thioredoxin Activity Fluorescent Assay. The subcellular localization of Trx-1, Trx-2 and TBP-2 was evaluated by cellular immunofluorescence. The cell viability was detected by Cell Counting Kit-8 (CCK-8) and the LC3-II protein level was detected to evaluate the autophagy level. The interaction between Trx-2 and TBP-2 was examined by Co-Immunoprecipitation (Co-IP). Results: The results showed that the mRNA levels of the Trx-1, Trx-2 and TBP-2 were kinetically changed after treatment with $50 \mu \mathrm{M}$ of $\mathrm{H} 2 \mathrm{O} 2$ for different duration. Exposure to $\mathrm{H} 2 \mathrm{O} 2$ increased the expression of Trx-2 and TBP-2 but not Trx-1, while the exposure inhibited Trx-1 activity. TBP-2 was co-localized with Trx-1 and Trx-2, exposure to $\mathrm{H} 2 \mathrm{O} 2$ enriched co-localization of TBP-2 to Trx-1 but not Trx-2, and increased the interaction between TBP-2 and Trx-1. Under normal circumstances, Trx-1 over-expression enhanced autophagic response and mainly regulates autophagy in the initiation. Conclusions: This study demonstrates differential roles of Trx-1 and Trx-2 in cellular response to oxidative stress, and oxidative stress increased Trx-1 interacting to TBP-2 and Trx-1 regulating autophagic response.

\section{Background}

Blindness because of cataracts afflicts millions of people worldwide, and continues to be the major cause of blindness globally [1]. Generally, cataracts can be classified as age-related cataracts, paediatric cataracts, and cataracts secondary to other causes. Age-related cataract is the most common type in adults, with the onset between age 45 years and 50 years, and is a multifactorial disease caused by interactions between genes (e.g., genetic polymorphisms susceptibility to age-related cataract) and environmental factors including aging, gender, ultraviolet rays, ionizing radiation and chemicals[2]. One of the mechanisms for the above interactions is that the process triggers oxidative damage to lens and further leads to age-related diseases including age-related cataract.

Substantial evidences point out aggravated oxidative stress as a vital factor for cataract formation[3]. The lens epithelial cells (LECs) are the most metabolically active cells of the lens, undergoing oxidation, insolubilisation, and crosslinking[4]. Previous studies indicated that exposure to environmental factors generate reactive oxygen species (ROS) and disrupt the redox status in LECs $[4,5]$. ROS-induced damage in the LECs may consist of oxidation of proteins, DNA damage, and/or lipid peroxidation, all of which have been implicated in cataractogenesis[6]. Oxidative stress, a major and ubiquitous stressing factor, was initially selected for investigating the cellular response to stress. Thus, elucidation on the oxidative stress response of LECs is critical to unveil oxidative and redox imbalance in the etiology of age-related cataract. 
The thioredoxin (Trx) system, which is composed of NADPH, thioredoxin reductase (TrxR), and thioredoxin, is a key antioxidant system in defense against oxidative stress through its disulfide reductase activity regulating protein dithiol/disulfide balance[7]. The Trx system provides the electrons to thiol-dependent peroxidases (peroxiredoxins) to remove reactive oxygen and nitrogen species with a fast reaction rate[8]. Trx is an important redox regulator with cytosolic Trx 1 and mitochondrial Trx 2 isozymes, and plays a key role in reduces a variety of substances to maintain the intracellular redox balance. As a negative regulator of the reducing capacities of Trx, thioredoxin binding protein-2 (TBP-2), which is initially known as Vitamin D3 Upregulated Protein 1 (VDUP1), binds with the reduced form of Trx and forms an intermolecular disulfide bond at the redox-active catalytic domain[9]. TBP-2 acts as a central regulator of cellular signaling pathways involved in the oxidative stress mechanism[9].

In human lens, our previous studies showed that high cellular levels of TBP-2 can potentially suppress Trx bioavailability and increase oxidation sensitivity, and overexpression of TBP-2 also causes slow growth by mitotic arrest and apoptosis[10]. Upon low level of oxidative stress, autophagic response initiated in LECs. TBP-2 positively regulates this response and reduces cell viability in LECs, through mediating autophagy in the initiation stage, which is mTOR-independent and probably caused by the dephosphorylation of Akt under oxidative stress[11]. However, it remains unclear on the role of Trx/TBP-2 in oxidative stress induced autophagy in LECs. Therefore, this study investigated the autophagic response of LECs induced by oxidative stress, and explored the possible mechanism of Trx/TBP-2 in autophagy regulation.

\section{Methods}

\section{Cell Culture and Treatment}

Human lens epithelial cells HLE-B3 (also named LECs), immortalized by infection with adenovirus 12SV40 (ATCC, USA), were grown and maintained in 1:1 Dulbecco's modified Eagle's medium and Ham's F12 medium (DMEM/F-12) (\#10-092-CV, Corning Cellgro, USA) which is supplemented with $10 \%$ fetal bovine serum (FBS) (Bioind, Israel), $100 \mathrm{U}$ each of penicillin and streptomycin (Gibco, USA) in humidified $\mathrm{CO}_{2}$ incubator. For overexpression of Trx-1, the LECs were transfected with Lipofectamine 2000 and vector-control (vector) or TBP-2-overexpression lentivirus (TBP-2), which were designed and constructed by Shanghai Genechem.

Cells were plated 24 hours before the experiments and experiments were performed when cell confluence reached $80 \%-90 \%$. Before the treatment, cells were washed with PBS twice to avoid the influence of serum. Cells were treated with $50 \mu \mathrm{M} \mathrm{H}_{2} \mathrm{O}_{2}$ in DMEM/F12 without serum for different durations.

According to our previous research[11], in wortmannin and bafilomycin A1 inhibitory experiments, cells were treated with complete medium or $50 \mu \mathrm{M}$ of $\mathrm{H}_{2} \mathrm{O}_{2}$ was treated for 12 hours. In these 12 hours, $10 \mu \mathrm{M}$ wortmannin (\#S2758, Selleck, USA) was added to the medium after six hours; 50nM bafilomycin A1 (\#BF1116, Sangon, China) was added at the ninth hour. 
Total RNAs were isolated from cells using Trizol (\#15596026, Invitrogen, USA). The reverse transcription (RT) was conducted by the PrimeScript ${ }^{\text {TM }}$ RT Master Mix (\#RR036A, Takara, Japan), and the products were subjected to real-time polymerase chain reaction (PCR) analysis using a TB Green ${ }^{\mathrm{TM}}$ Premix Ex Taq ${ }^{\mathrm{TM}}$ II (\#RR420A, Takara, Japan) with an Applied Biosystems7500 Fast Real-Time PCR System (Applied Biosystems). PCR primers specific for Trx-1, Trx-2, TBP-2 and beta-actin. were designed with the help of website - Primer bank[12] (Table 1). Dissociation curves of PCR products were obtained to guarantee amplification of the correct genes. Each sample was run in triplicate for technical repeats. The mean threshold cycle value of the triplicates and the $n$-fold difference in expression of the genes between different groups were determined after normalization to the expression level of beta-actin.

Table 1: The oligonucleotides served as the primers of qRT-PCR.

\begin{tabular}{lll}
\hline Gene symbols & Primers & \\
\hline beta-actin & Forward & 5'-CATGTACGTTGCTATCCAGGC-3' \\
& Reverse & 5'-CTCCTTAATGTCACGCACGAT-3' \\
Trx-1 & Forward & 5'-GTGAAGCAGATCGAGAGCAAG-3' \\
& Reverse & 5'-CGTGGCTGAGAAGTCAACTACTA-3' \\
Trx-2 & Forward & 5'-CTGGTGGCCTGACTGTAACAC-3' \\
& Reverse & 5'-TGACCACTCGGTCTTGAAAGT-3' \\
TBP-2 & Forward & 5'-AAGCTGTCCTCAGTCAGAGGCAAT-3' \\
& Reverse & 5'-ATGACTTTCTTGGAGCCAGGGACA-3' \\
\hline
\end{tabular}

\section{Protein extraction and Western Blot analysis}

The mitochondrial fraction was isolated according to the protocol of Mitochondria Isolation Kit for Cultured Cells (\#89874, Thermo, USA). Briefly, the cell pellets were vortexed in $800 \mu$ l of buffer A at medium speed for 5 seconds, and incubated on ice for exactly 2 minutes. Then tube was added in $10 \mu \mathrm{l}$ buffer $B$ to vortex at maximum speed for 5 seconds. After that, tube was incubated on ice for 5 minutes, vortexing at maximum speed every minute. $800 \mu \mathrm{l}$ buffer $\mathrm{C}$ was added in tube to mix, followed by centrifugation at $700 \mathrm{~g}$ for 10 minutes at $4^{\circ} \mathrm{C}$ to collect nuclei and membranes. The chilled supernatant after centrifugation was considered the cytosolic fraction and the sediment was the mitochondrial fraction. The supernatant was centrifuged at $12,000 \mathrm{~g}$ for 15 minutes at $4^{\circ} \mathrm{C}$. The supernatant was cytosol fraction, the pellet contained the isolated mitochondria. The sediment was resuspended in $500 \mu \mathrm{L}$ of buffer $\mathrm{C}$ and centrifuged again at $12,000 \mathrm{~g}$ for 5 minutes. The final sediment was lysed with $2 \%$ CHAPS 
in Tris-buffered saline. The subcellular fractions were validated by Western blot using cytosolic and mitochondria markers (See Supplementary Figure S1, Additional File 1).

The BCA Protein Assay Kit (\#23225, Thermo, USA) was used to determine the protein concentration. Same amounts of protein were subjected to SDS-PAGE, transferred to a polyvinylidene difluoride (PVDF) membrane (Millipore, USA) and blocked for 1 hour with $5 \%$ nonfat dry milk in Tris buffered saline (TBS-T, $10 \mathrm{mM}$ Tris- $\mathrm{HCl}[\mathrm{pH} 7.5], 100 \mathrm{mM} \mathrm{NaCl}$, and $0.1 \%$ Tween 20$)$. Primary antibody incubations were performed at the following dilutions: Trx-1, 1: 2000 (\#ab86255, Abcam, USA); Trx-2, 1: 100 (\#sc-133200, Santa Cruz Biotech, USA); Voltage-dependent Anion Channel (VDAC), 1: 5000 (\#ab15895, Abcam, USA); beta-tubulin, 1:3000 (\#EM1701-59, Huaan Biotechnology, China); TBP-2, 1: 200 (\#K0204-3, MBL, Japan); Microtubule-associated protein 1A/1B-light chain 3 (LC3), 1: 1000 (\#L7543, Sigma-Aldrich, USA); p62, 1: 1000 (\#8025, CST, USA); Glyceraldehyde 3-phosphate Dehydrogenase (GAPDH), 1: 5000 (\#M20006, Abmart, China). All incubations were done at $4^{\circ} \mathrm{C}$ overnight. Then, the membrane was washed thrice with TBS-T for 30 minutes and followed by respective secondary antibody incubations with goat antirabbit/mouse IgG-horseradish peroxidase (1:5000, Santa Cruz, USA). Immunodetection was performed with the ECL Western Blot Detection System (Millipore, USA). The immunoblot was analyzed with an

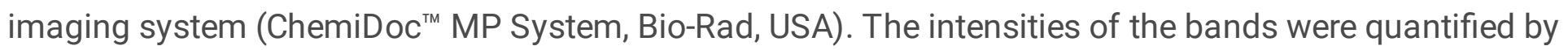
densitometry using Image Lab (Bio-Rad, USA). Values were reported as percentage of the control values \pm SEM.

\section{Trx-1 activity evaluation}

Trx-1 activity was evaluated by Thioredoxin Activity Fluorescent Assay Kit (\#11527, Cayman, USA). Briefly, Cell lysates was added in a certain volume of assay buffer, TrxR, beta-NAPDH and fluorescent substrate in sequence, followed by recording the emission at $545 \mathrm{~nm}$ after $520 \mathrm{~nm}$ excitation for 60 minutes in a fluorescent plate reader at room temperature. The obtained data was calculated according to the increasing fluorescence intensity for the time of the reaction within a liner range.

\section{Immunofluorescence staining $\square I F \bigotimes$}

Cells were seeded on cleaned coverslips 24 hours prior to the treatment. After $\mathrm{H}_{2} \mathrm{O}_{2}$ treatment, the cells were fixed in $4 \%$ formaldehyde solution. Next, the cells were permeabilized with $0.2 \%$ Triton-X-100 (SigmaAldrich, USA) and blocked with 20\% goat serum (Applygen, China). Subsequently, the cells were incubated with anti-Trx-1 (\#ab86255, Abcam, USA) or with anti-Trx-2 (\#ab185544, Abcam, USA) followed by antiTBP-2 (\#K0204-3, MBL, Japan) overnight at $4^{\circ} \mathrm{C}$. After thorough washing with PBS, Alexa Fluor ${ }^{\circledR} 488$ Goat Anti-Rabbit IgG (\#150077, Abcam, USA), Alexa Fluor® 488 Goat Anti-Mouse IgG (\#150113, Abcam, USA) or Alexa Fluor® 555 Goat Anti-Mouse IgG (\#ab150114, Abcam, USA) solution was used to incubate the cells for 1.5 hours at room temperature. The mitochondria were stained with MitoTracker ${ }^{\circledR}$ Deep Red FM (\#M22426, Thermo, USA). The nuclei were stained with DAPI. Eventually, the coverslips were mounted on the slides with a drop of mounting medium (H-1000, Vector, USA). Fluorescent images were captured with a Leica DM2500 Fluorescence microscope. 


\section{Cytotoxicity Assay}

Cytotoxicity assays were performed using a Cell Counting Kit-8 (CCK-8) assay (Dojindo, Japan). The cells were incubated with indicated concentrations of $\mathrm{H}_{2} \mathrm{O}_{2}$ dissolved in DMEM/F12 without serum for 12 or 24 hours. After the treatment, the medium was removed and the cells were washed with PBS. Then, each well was refilled with $90 \mu \mathrm{L}$ of DMEM/F12 supplemented with $10 \%$ fetal bovine serum and $10 \mu \mathrm{L}$ of CCK-8 reagents and incubated in $37^{\circ} \mathrm{C}$ for 3 hours. The cell viability was evaluated with $\mathrm{OD} 450$ values using a 96-well microplate reader (Bio-Rad, USA).

\section{Co-Immunoprecipitation}

Anti-TBP-2 antibody was used for the immunoprecipitation of TBP-2 and Trx-1 complex. The cytoplasm proteins were incubated with $2 \mu \mathrm{g}$ anti-TBP-2 (\#K0204-3, MBL, Japan) overnight at $4{ }^{\circ} \mathrm{C}$. Then the immunoprecipitated complexes was incubated with magnetic beads (Bio-Rad, USA) for 4 hours at $4{ }^{\circ} \mathrm{C}$, washed three times with ice-cold washing buffer, resuspended in electrophoresis SDS sample buffer, boiled for 5 min at $95{ }^{\circ} \mathrm{C}$, and analyzed on $10 \%$ SDS-PAGE. Immunoblotting was performed using the following primary antibodies: anti-TBP-2 (1:2000, \#ET1705-72, Huaan Biotechnology, China), anti-Trx1 (1:2000, for cytosol fraction; \#ab86255, Abcam, USA). The secondary antibodies used were as follows: goat anti-rabbit/mouse lgG-horseradish peroxidase (1:5000, Santa Cruz, USA)

\section{Statistics}

Three independent replications were performed, and the numerical data were presented as mean \pm standard error of mean (SEM). The differences between control and experimental groups were tested for significance with the independent Student's $t$-test or one-way ANOVA. The independent Student's $t$-test was used for comparison between the two groups, one-way ANOVA for multiple comparisons between the groups. A difference of $p<0.05$ was considered statistically significant.

\section{Results}

\section{Kinetic changes of $\operatorname{Tr} x-1, \operatorname{Tr} x-2$ and TBP-2 mRNA and protein levels upon oxidative stress}

In our previous study, we found that $50 \mu \mathrm{M} \mathrm{H}_{2} \mathrm{O}_{2}$ (low dose of oxidative stress) for $12 \mathrm{~h}$ induced autophagic response in LECs. To evaluate the role of Trx-1, Trx-2 and TBP-2 in response to oxidative stress in LECs, we firstly the mRNA and protein expression level of Trx-1, Trx-2 and TBP-2 under treatment by low concentration of $\mathrm{H}_{2} \mathrm{O}_{2}$ for different durations. RT-PCR analyses showed that exposure to $50 \mu \mathrm{M}$ of $\mathrm{H}_{2} \mathrm{O}_{2}$ for $12 \mathrm{~h}$ up-regulated TBP-2 transcription (Figure 1a). Exposure to $\mathrm{H}_{2} \mathrm{O}_{2}$ for $6 \mathrm{~h}$ significantly inhibited Trx-1 and Trx-2 transcription while extension of the exposure to 12 and $24 \mathrm{~h}$ enhanced Trx-2 but not Trx-1 transcription (Figure 1a). Western blot analyses showed that exposure to $50 \mu \mathrm{M}$ of $\mathrm{H}_{2} \mathrm{O}_{2}$ for $12 \mathrm{~h}$ increased protein expression level of TBP-2 and Trx-2, but not Trx-1 (Figure 1b). 
Since the protein expression level was not changed under oxidative stress, we examined the activity of Trx-1. The results showed that the activity of Trx-1 was significantly inhibited in LECs exposed to 25,50 or $100 \mu \mathrm{M}$ of $\mathrm{H}_{2} \mathrm{O}_{2}$ for $12 \mathrm{~h}$ (Figure 2). TBP-2 is a negative regulator of Trx-1. Thus, inhibition of Trx-1 activity might be due to increased protein expression of TBP-2 when the cells were exposed to $\mathrm{H}_{2} \mathrm{O}_{2}$ (Figure 1b).

\section{Co-localization between Trx-1 or Trx-2 and TBP-2}

To further investigate the relationship between Trx-1 or Trx-2 and TBP-2, we examined the cellular localization of Trx-1, Trx-2 and TBP-2. Without treatment of $\mathrm{H}_{2} \mathrm{O}_{2}$, Trx-1 was mainly localized in the nucleus, Trx-2 was mainly expressed in the cytoplasm, where Trx-2 was co-localized with mitochondria (See Supplementary Figure S2, Additional File 2), and TBP-2 was stayed in the cytoplasm but accumulated in perinuclear region (Figure 3). When the cells were treated by $50 \mu \mathrm{M}$ of $\mathrm{H}_{2} \mathrm{O}_{2}$ for $12 \mathrm{~h}$, the cellular localization of Trx-1 did not change obviously, but the TBP-2 was appeared in pan-cytoplasm and co-localized with Trx-1 (Figure 3a). However, under treatment of $\mathrm{H}_{2} \mathrm{O}_{2}$, Trx-2 was accumulated in the mitochondria, while the co-localization was increased between Trx-2 and TBP2 (Figure 3 and See Supplementary Figure S2, Additional File 2). Taken together, these results showed that in response to oxidative stress, TBP-2 was co-localized more with Trx-1 but not Trx-2.

\section{Interaction between $T r x-1$ and TBP-2}

Since Trx-1 was co-localized with TBP-2 in LECs under oxidative stress, we next explored the interaction between Trx-1 and TBP-2. Co-Immunoprecipitation assay showed that when TBP-2 was pulled down by anti-TBP-2 antibody, Trx-1 was detectable in the immunoprecipitated protein complex (Figure 4). When the cells were treated with $50 \mu \mathrm{M}$ of $\mathrm{H}_{2} \mathrm{O}_{2}$, Trx-1 was accumulated more in the pull-down immunoprecipitated protein complex compareing to that in control group.

\section{Autophagic regulation by $T r x-1$ in response to oxidative stress}

Our previous study showed low level treatment triggers autophagy. Here, we investigated the role of Trx-1 in oxidative stress induced autophagy. We firstly established Trx-1 stable overexpression (Figure 5). In Trx-1 stably expressed LECs, the ratio of LC3-II to LC3-I was significantly increased, suggesting Trx-1 overexpression induces autophagy. Furthermore, induction of autophagy by Trx-1 inhibited LC3 protein response of the cells to $50 \mu \mathrm{M}$ of $\mathrm{H}_{2} \mathrm{O}_{2}$ treatment (Figure 6a). Similar response of p62 protein was found when Trx-1 stably expressed cells were treated by $\mathrm{H}_{2} \mathrm{O}_{2}$ (Figure 6a). Meanwhile, cytotoxicity by oxidative stress was attenuated in Trx-1 over-expressed cells (Figure 6b).

To further confirm autophagic regulation by Trx-1 in response to oxidative stress, the autophagic inhibitors bafilomycin A1 and wortmannin were applied to cells. The results showed that Trx-1 overexpression enhanced bafilomycin A1 induced LC3-II aggregation (Figure 7a). This cellular response was also found in the Trx-1 stably expressed cells under oxidative stress (Figure 7a). On the other hand, wortmannin treatment could abolished Trx-1 over-expression induced autophagy (Figure 7b). 


\section{Discussion And Conclusion}

In the present study, high concentration of $\mathrm{H}_{2} \mathrm{O}_{2}$ caused apoptosis in LECs[10], low intensity of $\mathrm{H}_{2} \mathrm{O}_{2}$ induced cellular impairment model was applied to explore molecular mechanism of oxidative stress induced autophagic response in LECs[11]. We found that oxidative stress induced kinetic changes of Trx1, Trx-2 and TBP-2 transcription, increased the expression of Trx-2 and TBP-2 but inhibited Trx-1 activity, enriched co-localization of TBP-2 to Trx-1 but not Trx-2, and increased the interaction between TBP-2 and Trx-1. Furthermore, low intensity of oxidative stress enhanced Trx-1 induced autophagic response.

In this study, we found that while exposing to low concentration $\mathrm{H}_{2} \mathrm{O}_{2}$, the transcription of $\operatorname{Trx}-1$ and Trx-2 decreased at $6 \mathrm{~h}$, but began to rise at $12 \mathrm{~h}$ and $24 \mathrm{~h}$. It is interesting that the transcription of Trx-2 enhanced but not Trx-1 transcription at 24h compared to the control group. Dafre et al. [13] predicted that methylglyoxal (MGO), a saccharifier that activates nuclear factor-erythroid 2-related factor 2 (Nrf2) which is the transcription of Trx-1, could promote the expression of Trx-1. On the contrary, the expression of Trx1 decreased under apoptosis induced by MGO in endothelial cells; they believed that the reason was that MGO broken the expression of Trx-1 induced by Nrf2. Previous studies have found that autophagy is induced by the treatment of $50 \mu \mathrm{M} \mathrm{H}_{2} \mathrm{O}_{2}$ for 12 hours. Therefore, we supposed that the decrease of $\mathrm{Trx}$ mRNA level is most likely due to the influence of $\mathrm{H}_{2} \mathrm{O}_{2}$ on the transcription of Trx at first $6 \mathrm{~h}$. At $12 \mathrm{~h}$, autophagy may act as a protective role to rescue the transcription of Trx. The specific mechanisms involved need to be further studied in the future.

Both cytosolic Trx-1 and mitochondrial Trx-2 isozymes have multiple physiological functions in cells, and their bioavailability are negatively controlled through active-site binding to TBP-2. Thus, balance between TBP-2 and Trx plays a key role in oxidative and redox biological process. Our previous study showed that high cellular levels of TBP-2 can potentially suppress Trx bioavailability and increase oxidation sensitivity[10]. The present study demonstrated differential roles of Trx-1 and Trx-2 in cellular response to oxidative stress, which extend our knowledge on Trx/TBP-2 axis upon low intensity of oxidative stress.

Autophagy maintains cellular homeostasis through degradation of intracellular proteins, lipids, and organelles in response to various environmental conditions, including nutritional starvation[14], endoplasmic reticulum (ER) stress[15], increased oxidative stress[16], and senescence[17]. Our previous study showed oxidative stress induced autophagic response of LECs with increased LC3-II, p62, and GFP/mCherry-LC3 puncta. Overexpression of TBP-2 further strengthens autophagic response in the initiation stage, which is mTOR-independent and probably caused by the dephosphorylation of Akt under oxidative stress[18]. In the present study, we used the autophagic inhibitor bafilomycin A1 to block the its last stage which is the fusion of autophagosomes and lysosomes and autophagic inhibitor wortmannin to inhibit autophagy at the initiation stage. Our data indicated that Trx-1 mainly regulates autophagy in the initiation stage and facilitates autophagic induction, which consistent with our previous finding on the autophagic flux induction by TBP-2 in the initiation stage[18]. 
Age-related cataracts still remain the leading cause of blindness in middle-income and low-income countries[19]. With advancements in technology, surgical techniques, instrumentation, design of intraocular lenses, and medications, cataract surgery has been shown to be a safe and effective intervention to improve vision and quality of life $[2,20]$. However, cataract surgery was very limited to large number of age-related cataract patients in general population. Thiol repair systems were damaged in all types of cataracts, especially in age-related cataracts[21]. Our findings shed light on the mechanism of Trx/TBP2 under oxidative stress and the protective role of Trx-1 in oxidative stress induced impairment of human LECs, suggesting a preventive and therapeutic targets for age-related cataracts.

In conclusion, this in vitro study differential roles of Trx-1 and Trx-2 in cellular response to oxidative stress, and the distinct role of Trx-1 in oxidative stress induced autophagic response through interaction with TBP-2. Further in vivo studies await to confirm the role Trx/TBP-2 axis against oxidative stress during the cataract development.

\section{Abbreviations}

TBP-2: thioredoxin binding protein-2

Trx: thioredoxin

LECs: Human lens epithelial cells

CCK-8: Cell Counting Kit-8

Co-IP: Co-Immunoprecipitation

ROS: reactive oxygen species

TrxR: thioredoxin reductase

VDUP1: Vitamin D3 Upregulated Protein 1

FBS: fetal bovine serum

DMEM/F-12: Dulbecco's modified Eagle's medium and Ham's F-12 medium

RT: reverse transcription

PCR: polymerase chain reaction

PVDF: polyvinylidene difluoride

VDAC: Voltage-dependent Anion Channel

TBS-T: Tris buffered saline- Tween 20 
LC3: Microtubule-associated protein 1A/1B-light chain 3

GAPDH: Glyceraldehyde 3-phosphate Dehydrogenase

IF: Immunofluorescence staining

MGO: methylglyoxal

Nrf2: nuclear factor-erythroid 2-related factor 2

ER: endoplasmic reticulum

\section{Declarations}

Ethics approval and consent to participate

Not applicable.

Consent for publication

Not applicable.

Availability of data and materials

All data generated or analyzed during this study are included in this published article [and its supplementary information files].

Competing interests

The authors declare that they have no competing interests.

Funding

This work was supported by the National Natural Science Foundation of China [Grant no. 81470612 and 81670832].

Authors' contributions

$\mathrm{JHH}$ and LFS were major contributors in doing experiments and writing the manuscript. CSZ and KY contributed to the literature search and preparation of the manuscript and figures. YBY was responsible for the design of the research. All authors read and approved the final manuscript.

Acknowledgements

The authors thank Guangdi Chen for his assistance in writing. 


\section{References}

1. Lee $\mathrm{CM}$, Afshari NA. The global state of cataract blindness. Current opinion in ophthalmology. 2017;28(1):98-103.

2. Liu YC, Wilkins M, Kim T, Malyugin B, Mehta JS. Cataracts. Lancet (London, England). 2017;390(10094):600-612.

3. Liu XF, Hao JL, Xie T, Malik TH, Lu CB, Liu C et al. Nrf2 as a target for prevention of age-related and diabetic cataracts by against oxidative stress. Aging cell. 2017;16(5):934-942.

4. Vinson JA. Oxidative stress in cataracts. Pathophysiology : the official journal of the International Society for Pathophysiology. 2006;13(3):151-162.

5. Babizhayev MA. Mitochondria induce oxidative stress, generation of reactive oxygen species and redox state unbalance of the eye lens leading to human cataract formation: disruption of redox lens organization by phospholipid hydroperoxides as a common basis for cataract disease. Cell biochemistry and function. 2011;29(3):183-206.

6. Babizhayev MA, Yegorov YE. Reactive Oxygen Species and the Aging Eye: Specific Role of Metabolically Active Mitochondria in Maintaining Lens Function and in the Initiation of the Oxidation-Induced Maturity Onset Cataract--A Novel Platform of Mitochondria-Targeted Antioxidants With Broad Therapeutic Potential for Redox Regulation and Detoxification of Oxidants in Eye Diseases. American journal of therapeutics. 2016;23(1):e98-117.

7. Lourenco Dos Santos S, Petropoulos I, Friguet B. The Oxidized Protein Repair Enzymes Methionine Sulfoxide Reductases and Their Roles in Protecting against Oxidative Stress, in Ageing and in Regulating Protein Function. Antioxidants (Basel, Switzerland). 2018;7(12).

8. Lu J, Holmgren A. The thioredoxin antioxidant system. Free radical biology \& medicine. 2014;66:7587.

9. Spindel ON, World C, Berk BC. Thioredoxin interacting protein: redox dependent and independent regulatory mechanisms. Antioxidants \& redox signaling. 2012;16(6):587-596.

10. Yu Y, Xing K, Badamas R, Kuszynski CA, Wu H, Lou MF. Overexpression of thioredoxin-binding protein 2 increases oxidation sensitivity and apoptosis in human lens epithelial cells. Free radical biology \& medicine. 2013;57:92-104.

11. Zhou J, Yao K, Zhang Y, Chen G, Lai K, Yin H et al. Thioredoxin Binding Protein-2 Regulates Autophagy of Human Lens Epithelial Cells under Oxidative Stress via Inhibition of Akt Phosphorylation. Oxidative medicine and cellular longevity. 2016;2016:4856431.

12. PrimerBank. https://pga.mgh.harvard.edu/primerbank/.

13. Dafre AL, Goldberg J, Wang T, Spiegel DA, Maher P. Methylglyoxal, the foe and friend of glyoxalase and Trx/TrxR systems in HT22 nerve cells. Free radical biology \& medicine. 2015;89:8-19.

14. He L, Zhang J, Zhao J, Ma N, Kim SW, Qiao S et al. Autophagy: The Last Defense against Cellular Nutritional Stress. Advances in nutrition (Bethesda, Md). 2018;9(4):493-504. 
15. Yorimitsu T, Klionsky DJ. Endoplasmic reticulum stress: a new pathway to induce autophagy. Autophagy. 2007;3(2):160-162.

16. Filomeni G, De Zio D, Cecconi F. Oxidative stress and autophagy: the clash between damage and metabolic needs. Cell death and differentiation. 2015;22(3):377-388.

17. Gewirtz DA. Autophagy and senescence: a partnership in search of definition. Autophagy. 2013;9(5):808-812.

18. Hu J, Yu Y. The Function of Thioredoxin-Binding Protein-2 (TBP-2) in Different Diseases. Oxidative medicine and cellular longevity. 2018;2018:4582130.

19. Flaxman SR, Bourne RRA, Resnikoff S, Ackland P, Braithwaite T, Cicinelli MV et al. Global causes of blindness and distance vision impairment 1990-2020: a systematic review and meta-analysis. The Lancet Global health. 2017;5(12):e1221-e1234.

20. Olson RJ. Cataract Surgery From 1918 to the Present and Future-Just Imagine! American journal of ophthalmology. 2018;185:10-13.

21. Wei M, Xing KY, Fan YC, Libondi T, Lou MF. Loss of thiol repair systems in human cataractous lenses. Investigative ophthalmology \& visual science. 2014;56(1):598-605.

\section{Additional File Legends}

\section{Additional File 1:}

Supplementary Figure S1: Mitochondrial purity is great. VDAC is peculiar to mitochondria, beta-tubulin only exists in cytoplasm

\section{Additional File 2:}

Supplementary Figure S2: Mitochondria was co-localized with Trx-1 or TBP-2.

Flowchart 1. A flowchart of the experimental scheme.

\section{Figures}


A
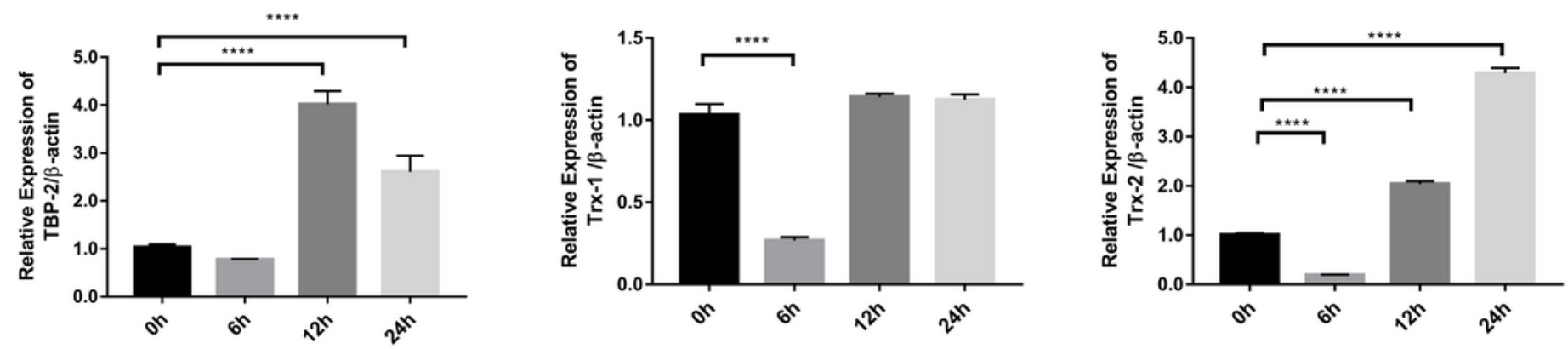

B
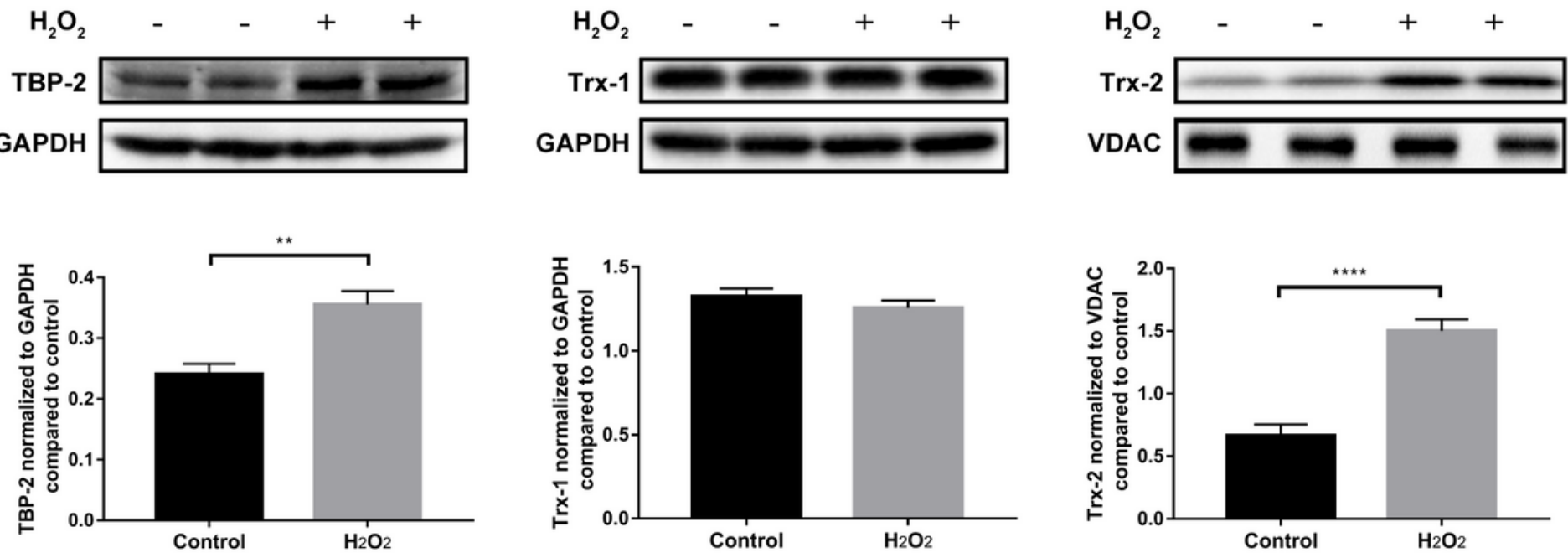

\section{Figure 1}

Kinetic changes of Trx-1, Trx-2 and TBP-2 mRNA and protein levels upon oxidative stress. (a) When cells were treated with $50 \mu \mathrm{M}$ of $\mathrm{H} 2 \mathrm{O} 2$ for various time durations, RT-PCR showed the change of Trx-1, Trx-2 and TBP-2 mRNA. (b) After treatment with $\mathrm{H} 2 \mathrm{O} 2(50 \mu \mathrm{M})$ for 12 hours, western blot revealed the protein expression level of Trx-1, TBP-2 and Trx-2. Data were presented as mean \pm SEM of three independent experiments. $* * P<0.01, * * * * P<0.0001$.

A

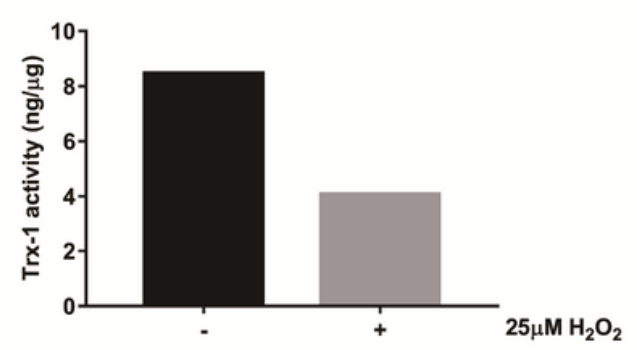

B

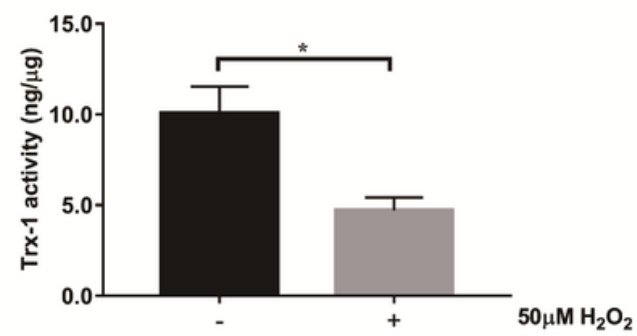

C

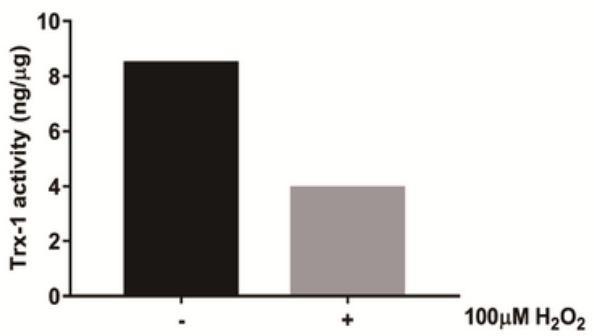

Figure 2 
Inhibition of Trx-1 under various concentration of $\mathrm{H} 2 \mathrm{O} 2$ for $12 \mathrm{~h}$. Data were presented as mean $\pm \mathrm{SEM}$ of three independent experiments. $* P<0.05$.

A
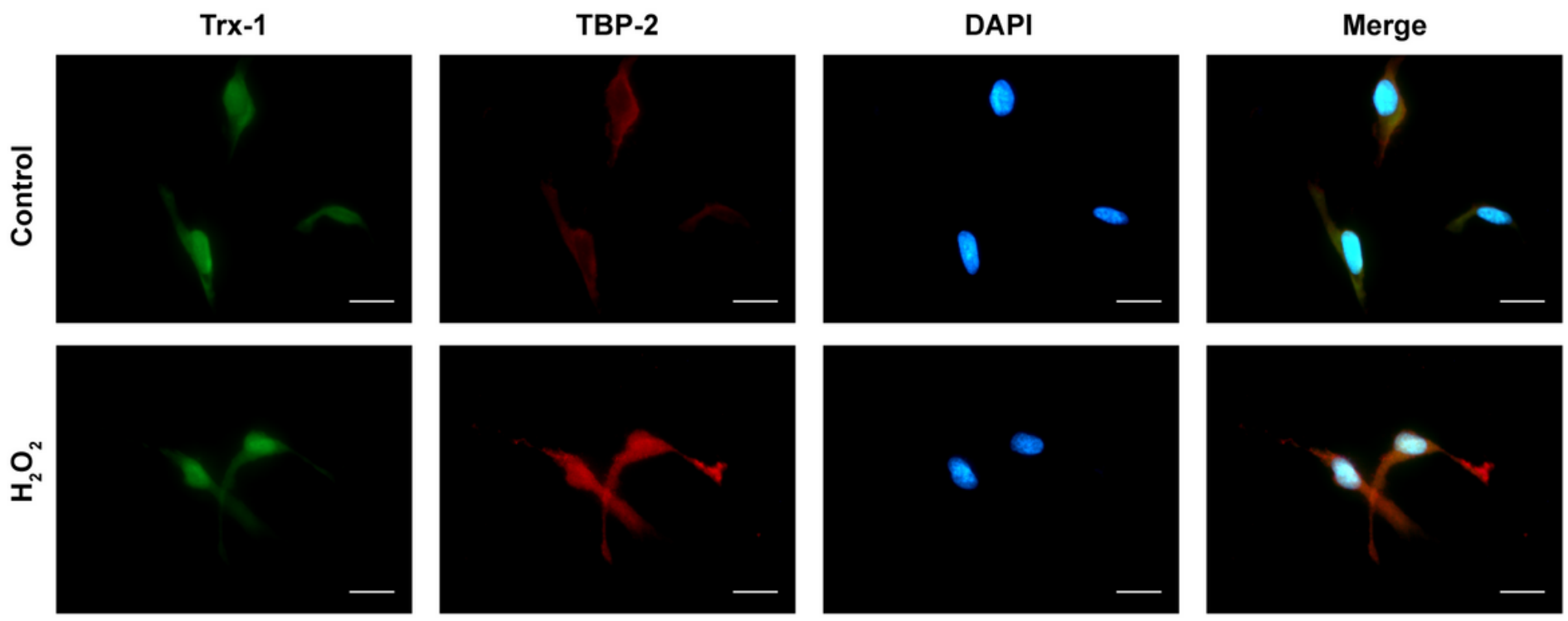

B

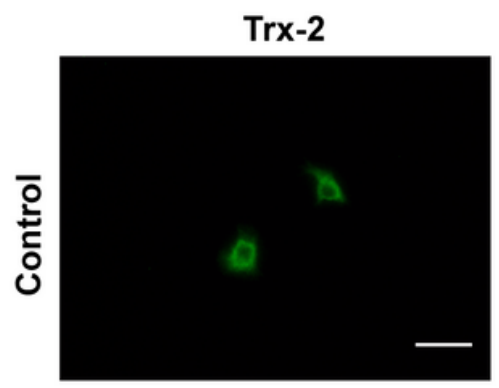

TBP-2
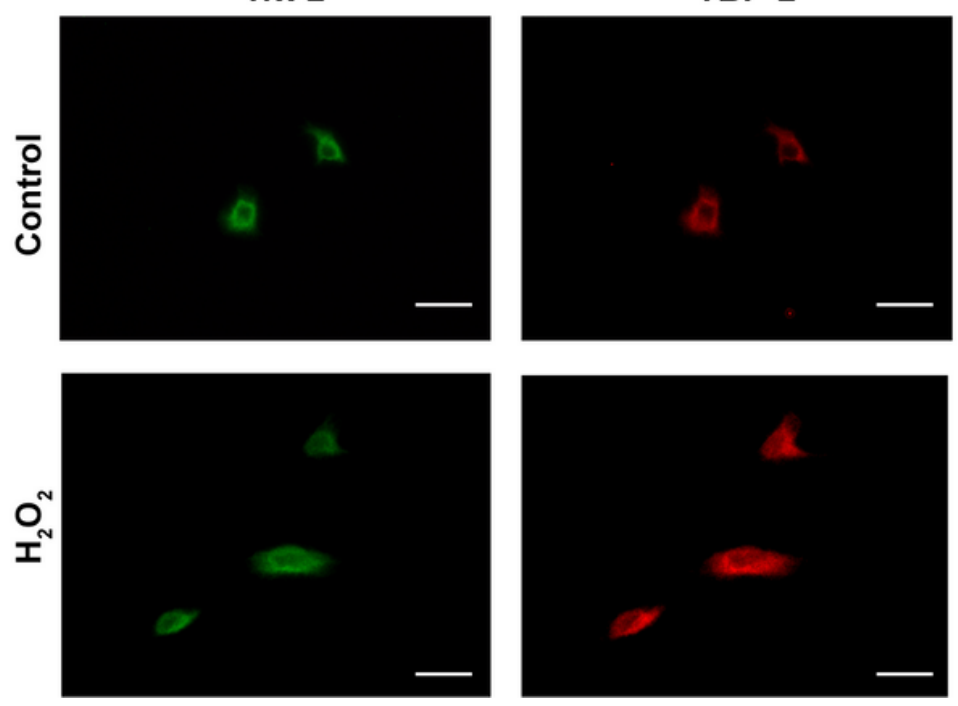

DAPI
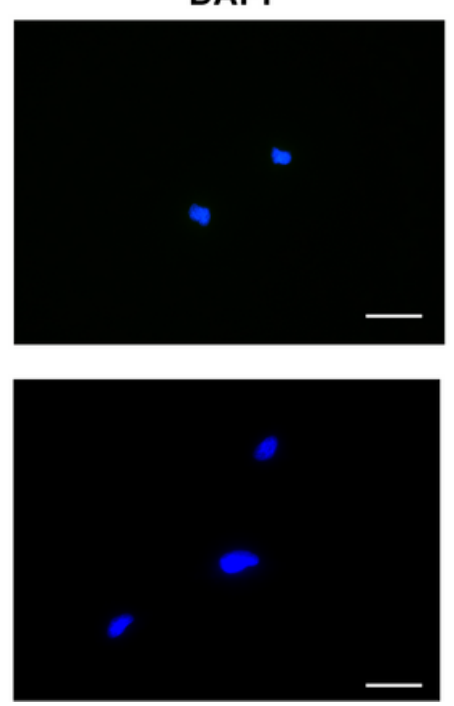

Merge
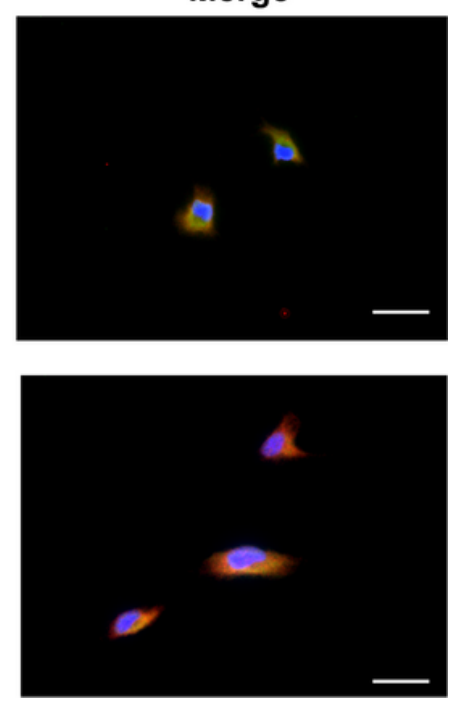

Figure 3

Under treatment of H2O2, TBP-2 co-localized with Trx-1, Trx-2. (a) The co-localization between Trx-1 and TBP-2. (b) The co-localization between Trx-2 and TBP-2. Scale bar, $25 \mathrm{~nm}$. 

$\mathrm{H}_{2} \mathrm{O}_{2}$
$+$
-
$+$

Anti-TBP-2

$+$

$+$

$\lg \mathbf{G}$

$-\quad-$

$+$

$+$

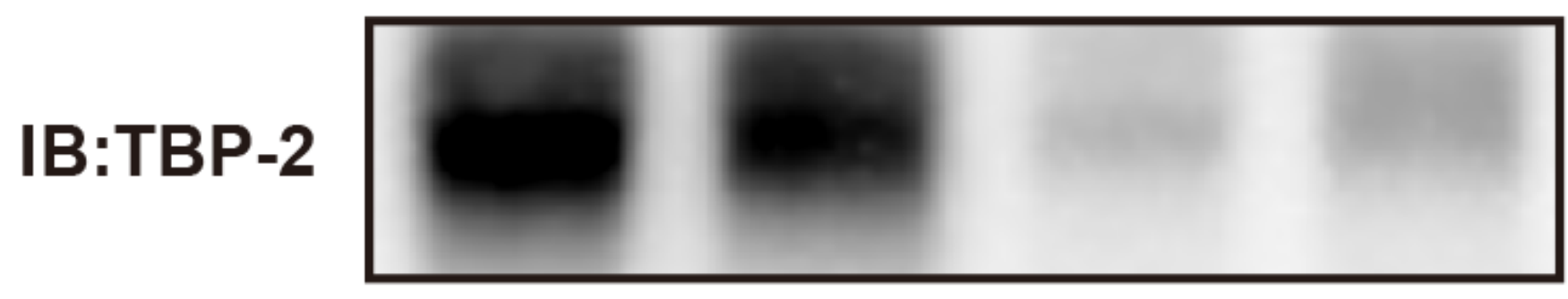

IB: Trx-1

Figure 4

The interaction between Trx-1 and TBP-2 in LECs under low oxidative stress. 
A

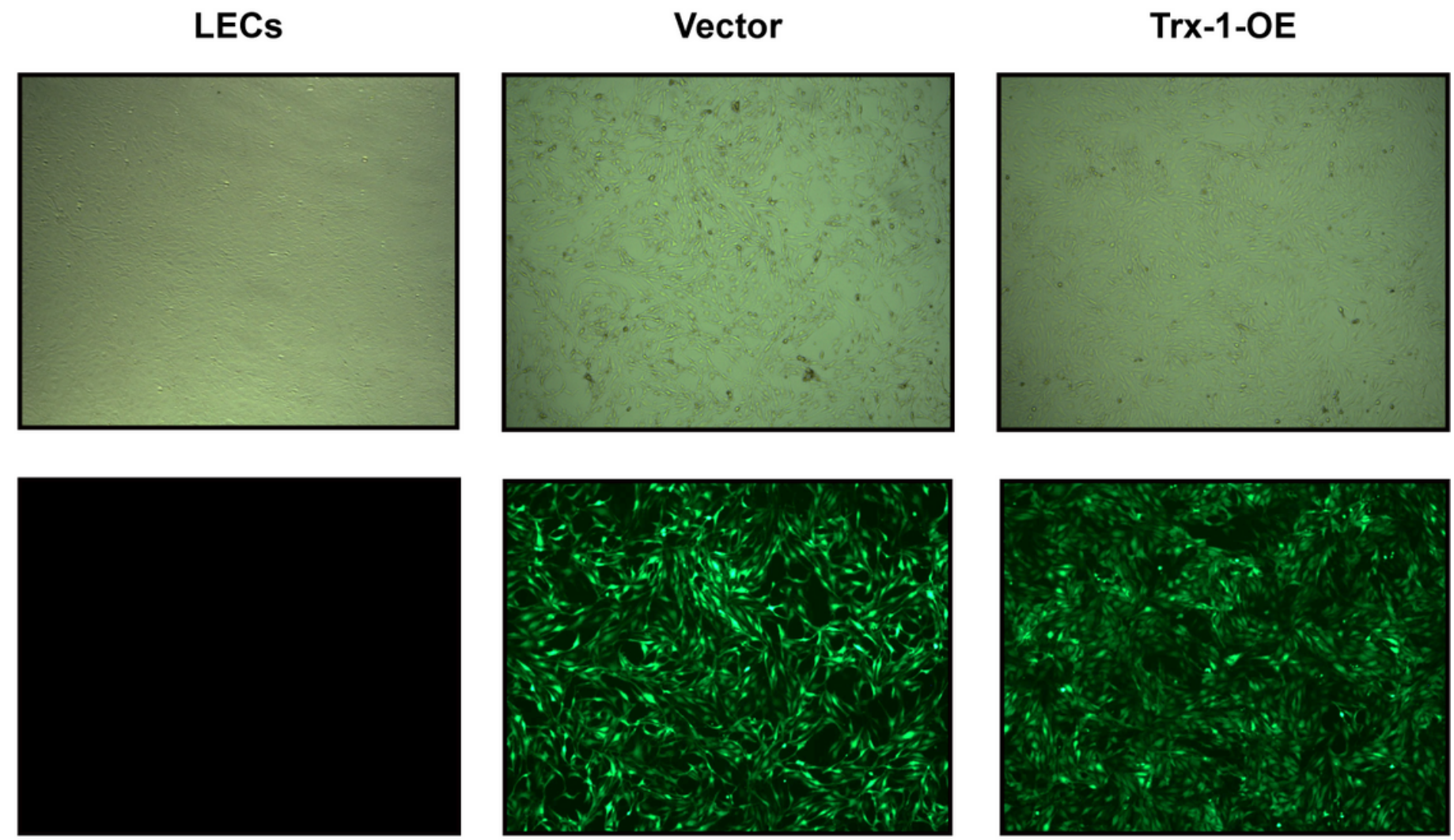

B

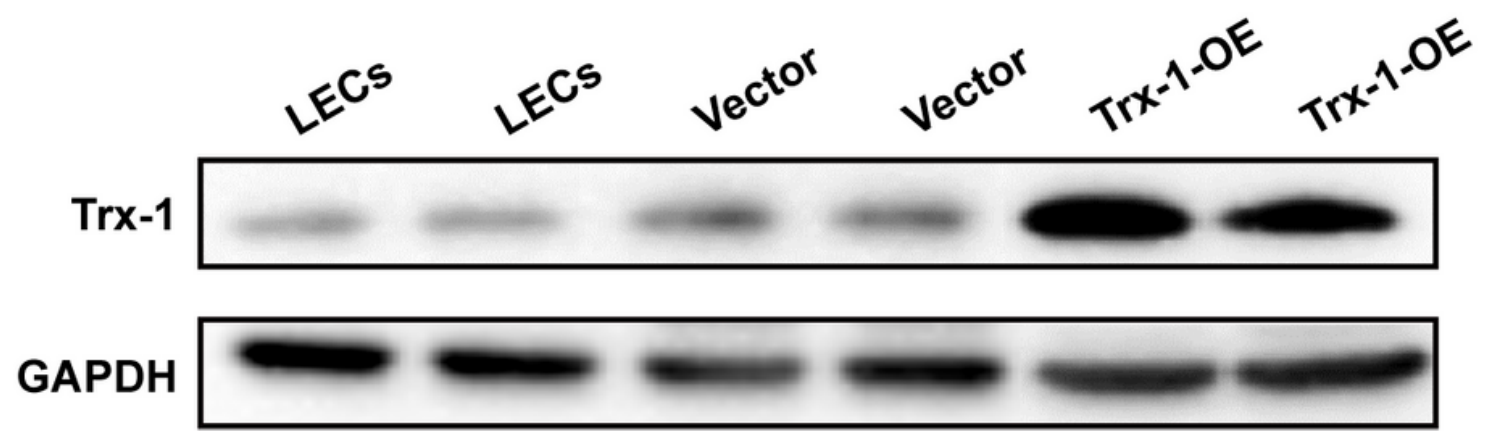

Figure 5

The verification of overexpression of Trx-1. (a) Fluorescent microscopy showed a high transfection efficiency. (b) There are the increase of Trx-1 level in overexpressed Trx-1 cell. 
A
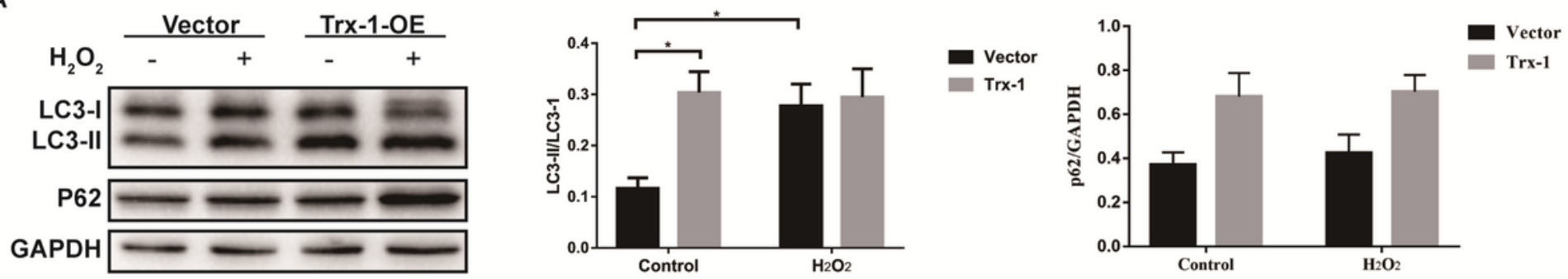

B
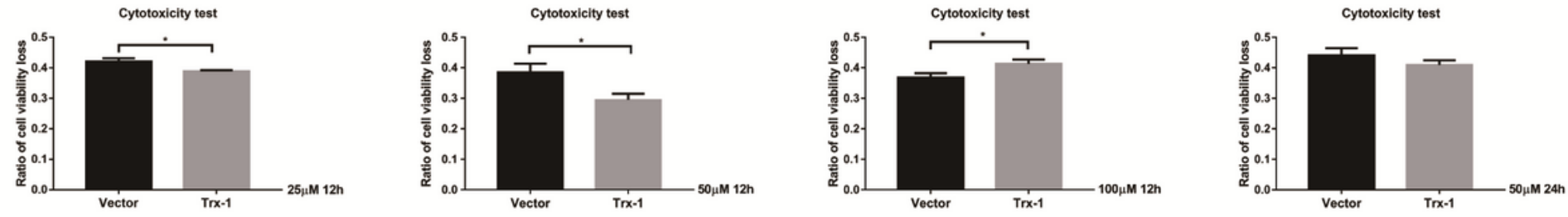

Figure 6

Autophagic regulation by Trx-1 in response to oxidative stress. (a) Overexpression of Trx-1 increased the level of intrinsic autophagy, but not the autophagy under oxidative stress. (b) Overexpression of Trx-1 weakened cytotoxicity under oxidative stress. Data were presented as mean \pm SEM of three independent experiments. $* P<0.05$. 
A

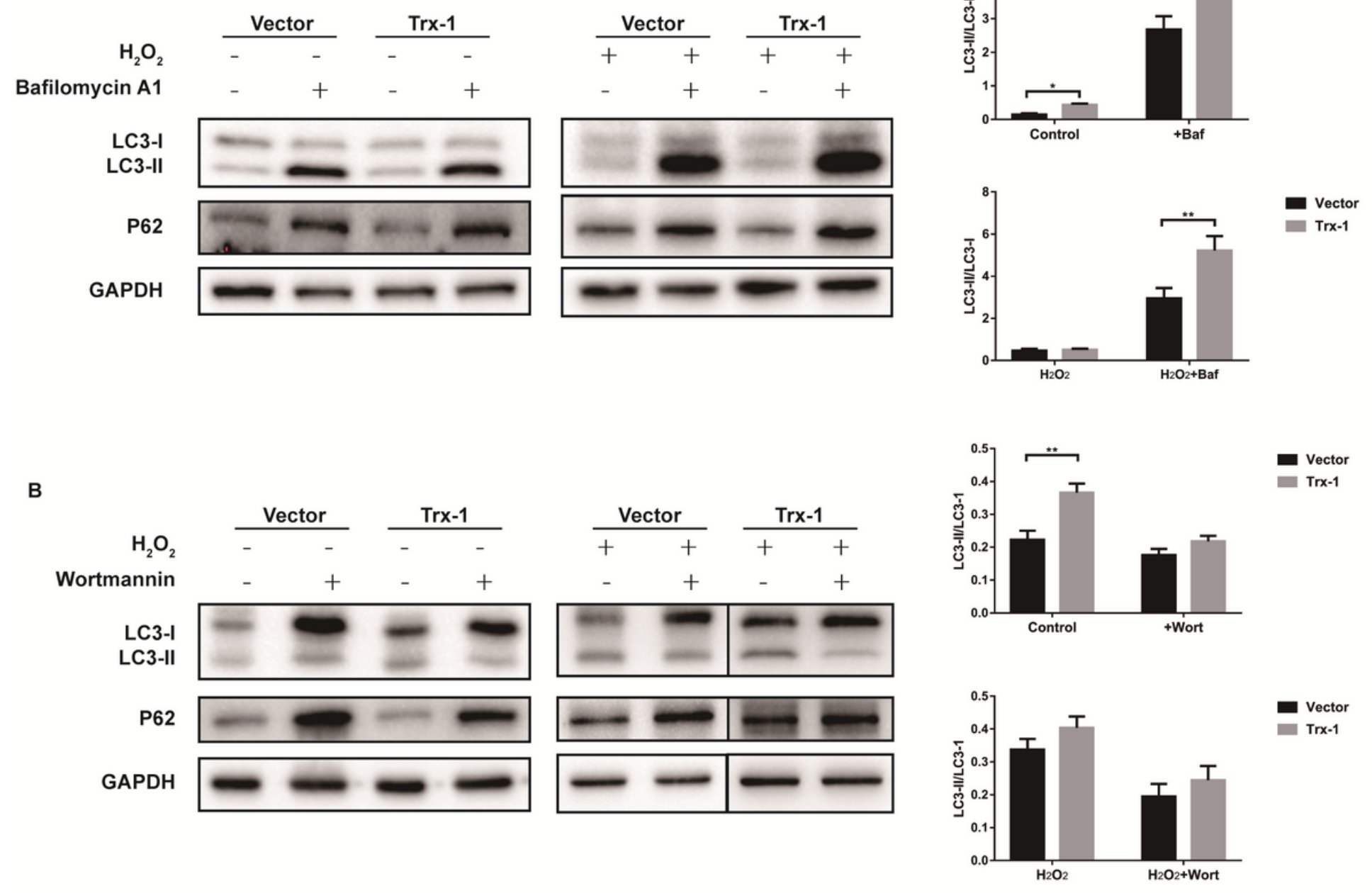

\section{Figure 7}

Trx-1 mostly regulates autophagy in the initiation stage. (a) In wortmannin group, Trx-1 cells showed significantly higher ratio of LC3-II/LC-I than Vector cells. (b) In bafilomycin A1 group, the ratio of LC3-II/LCI in both types of cells decreased. $* P<0.05, * * \mathrm{P}<0.01$.

\section{Supplementary Files}

This is a list of supplementary files associated with this preprint. Click to download.

- SupplementaryFigure1.tif

- SupplementaryFigure2.tif 
- Flowchart1.jpg

Page 19/19 